\title{
A thin target scheme for the Muon source
}

\author{
Alain Blondel ${ }^{\mathrm{a}}$, Jean Paul Fabre ${ }^{\mathrm{b}}$, Simone Gilardoni ${ }^{\mathrm{c}}$, \\ Nikolaos Vassilopoulos ${ }^{b}$ \\ ${ }^{a}$ LPNHE, Ecole Polytechnique, 91128 Palaiseau Cedex, France \\ ${ }^{\mathrm{b}}$ Cern, 1211, Geneva 23, Switzerland \\ ${ }^{\mathrm{c}}$ INFN Milano and Politecnico di Milano, Milano, Italy
}

\begin{abstract}
A pion production source using a thin target and a recirculating storage ring is considered. The potential advantages of the scheme are: i) the thin target could allow a better collection efficiency; ii) the proton beam dump is avoided; iii) light targets could be used. The disadvantages are: i) the high beam current stored in the recirculator, ii) constraints on timing. This scheme could be used for the Neutrino Factory but not for Muon Colliders.
\end{abstract}

The purpose of this paper is to perform a preliminary analysis of a thin target and recirculator scheme for the pion source of a muon machine such as a Neutrino Factory. A sketch of this is shown in Figure 1. This is similar to studies performed for antiproton sources (1).

Protons incoming from the source (i.e. a linac or a synchrotron) interact with a thin target and produce pions. Most protons do not interact and are captured

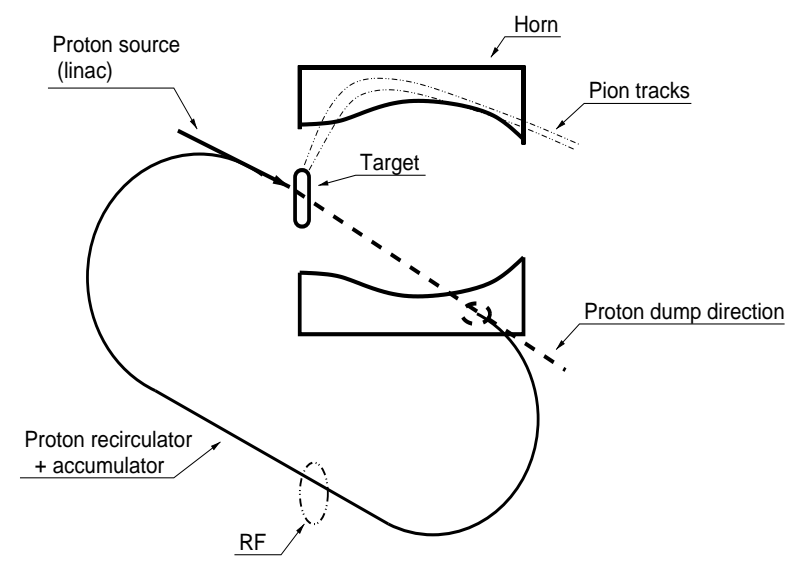

Fig. 1. Schema for the recirculating scenario 
in a recirculator which sends them back onto the target for several turns. There is no need for a dump, since all the protons produced by the injector are eventually absorbed in the target. The target can thus be thin (at limit point-like) and composed of low-Z materials which have the advantage of not producing heavy radioactive nuclei. This scheme could also be considered with an $\mathrm{H}^{-}$linac, the target acting in this case as a strip foil.

This scheme leads to a number of questions: i) will multiple scattering in the target increase the emittance so much that cooling of the protons should be necessary? ii) the energy deposited in the target is very localised and could become unbearable; iii) the accumulated current in the recirculator might be too high, iv) with the protons recirculating, the time structure of the pion beam acquires a structure at about one microsecond. Although these implications will not find here an answer in the context of a consistent scenario of a Neutrino Factory, they are addressed in turn.

\section{Multiple scattering}

The proton beam is spread by multiple scattering according to a characteristic angle $\theta_{0}$ :

$$
\theta_{0}=\frac{13.6(\mathrm{GeV})}{\beta c p(G e V)} \sqrt{\frac{x}{X_{0}}}(1+\log \text { terms })
$$

where $X_{0}$ is the radiation length for the given material (see ref. (2)), $x$ is the target thickness and $p$ the momentum of the incoming protons. This will

add up to the angular spread of the incident proton beam, $\sigma_{\theta}{ }^{\text {initial }}=\sqrt{\varepsilon^{\text {in }} / \beta}$ where $\varepsilon$ is the emittance of the beam and $\beta$ the optical focusing function at the target.

After one passage in the target the proton beam has a total angular spread which is the quadratic sum of these two contributions.

$$
\sigma_{\theta, \text { out }}^{2}=\frac{\varepsilon^{i n}}{\beta}+\theta_{0}^{2}
$$

and the corresponding transverse emittance is:

$$
\varepsilon^{\text {out }}=\varepsilon^{\text {in }}+\beta \theta_{0}^{2}
$$

At each turn, the fraction of protons that will interact, produce secondary particles and disappear from the beam is $a=\frac{x}{X_{I}}$, where $x$ is the thickness of 
the target, and $X_{I}$ is the interaction length for the given material. A typical proton will thus pass through the target $\frac{X_{I}}{x}$ times. (e.g. for a target composed by $x=1 \mathrm{~cm}$ of Beryllium which has $X_{I}=40 \mathrm{~cm}$ a proton passes on average 40 times), and the average emittance will then be $\varepsilon^{\text {out }}=\varepsilon^{i n}+\beta \theta_{0}^{2} \times \frac{X_{I}}{x}$.

A similar result is obtained if one fills the recirculator continuously. Let us now assume that the recirculation time is matched to the timing of the proton injector, as would be the case if the target also acted as an $\mathrm{H}^{-}$strip foil. At every turn a number $n 0$ of protons is added to the bunch, with an emittance equal to $\varepsilon^{i n}$. The remaining fraction $(1-a)$ of the proton beam is assumed to be recovered by the recirculation system. After infinite number of passages, the total angular divergence of the beam tends to:

$$
\varepsilon^{\text {equil. }}=\frac{n_{0} \sum_{0}^{\infty} \varepsilon^{i n} p_{k}}{n_{0} \sum_{0}^{\infty} p_{k}}+\frac{n_{0} \sum_{0}^{\infty} k \beta \theta_{0}^{2} p_{k}}{n_{0} \sum_{0}^{\infty} p_{k}}
$$

where $n_{0} p_{k}$ is the number of particles remaining after $k$ turns, with $p_{k}=$ $(1-a)^{k}$. With little algebra equation 4 becomes

$$
\varepsilon^{\text {equil. }}=\varepsilon^{i n}+\beta \theta_{0}^{2} \frac{1-a}{a}
$$

Playing with the definition of $\theta_{0}^{2}$ and $a$, the equilibrium beam emittance can be expressed as a function of the interaction and radiation lengths of the target material:

$$
\varepsilon^{\text {equil. }}=\varepsilon^{i n}+\beta \alpha_{0}^{2} \frac{X_{I}}{X_{0}}
$$

where $\alpha_{0}^{2}=\frac{13.6}{\beta c p}$; for a $2 \mathrm{GeV}$ beam (kinetic energy) $\alpha_{0}=5$ mrad.

For a given material, the emittance is a function only of the optical function $\beta$ at the target. One can then calculate the value $\beta^{\text {crit }}$ that corresponds to any limiting emittance. As an example we give in the table below the values of $\beta^{\text {crit }}$ for which $\varepsilon^{\text {equil. }}=2 \times \varepsilon^{\text {in }}$ for the linac design presented in ref. (5) where the beam emittance is given to be $\varepsilon^{i n}=0.6 \mu \mathrm{m}$.

\begin{tabular}{ccccc}
\hline Mat. & $X_{I}\left(\mathrm{~g} / \mathrm{cm}^{2}\right)$ & $X_{0}\left(\mathrm{~g} / \mathrm{cm}^{2}\right)$ & $\frac{X_{I}}{X_{0}}$ & $\beta^{\text {crit }}(\mathrm{cm})$ \\
\hline \hline$D_{2}$ & 54 & 186 & 0.43 & 5.6 \\
\hline$H_{2} O$ & 85 & 36 & 2.36 & 1 \\
\hline$B e$ & 75 & 65 & 1.15 & 2.1 \\
\hline$P b(\approx H g)$ & 194 & 6.4 & 30.3 & 0.08 \\
\hline
\end{tabular}


The best is clearly to chose a material with a short interaction length $\left(X_{I}\right)$ and a long radiation length $\left(X_{0}\right)$. The target thickness could then as long as a few centimetres and remain shorter than $\beta^{\text {crit }}$.

\section{$2 \quad$ Energy loss}

Another constraint for the target choice is given by the energy deposition in the target which is, as a first approximation, due to ionization and can be computed using $\frac{d E}{d x}$ for the given material. The energy deposited by secondary particles is presumably very small, since only a small fraction of the protons interact, and the target is too thin for showers to develop. For one proton, the energy loss at each passage is given by $x \frac{d E}{d x}$. The recirculator must therefore be equipped with a modest amount of re-accelerating devices (typically a few $\mathrm{MeV})$.

Since the average total path that every proton eventually undergoes in the target is one interaction length, the total energy loss is given by $P_{l o s s}=\frac{d E}{d x} X_{I}$ for each proton. This is calculated in the following table and should be compared with the proton energy. For heavy nuclei such as lead the loss is around $10 \%$ of the incoming power while for light materials this is kept around 5\%. This is clearly smaller than for a total absorption target, where a larger fraction (20-30\%) of the incident power is absorbed in the target itself.

\begin{tabular}{ccc}
\hline Mat. & $\frac{d E}{d x}(\mathrm{MeV}) \times X_{I}$ & \% of incoming beam energy for $2 \mathrm{GeV}$ protons \\
\hline \hline $\mathrm{D}_{2}$ & 112 & $5.5 \%$ \\
\hline $\mathrm{H}_{2} \mathrm{O}$ & 169 & $8.5 \%$ \\
\hline $\mathrm{Be}$ & 119 & $6 \%$ \\
\hline $\mathrm{Pb}$ & 217 & $11 \%$ \\
\hline
\end{tabular}

From this point of view again, low-Z materials show a non-negligible advantage over heavy materials. Moreover, the above power loss is computed for a proton beam energy of $2 \mathrm{GeV}$ but, if one considered higher energies (8 GeV to 32 $\mathrm{GeV}$ ), for a given total beam power, the power loss in the target would be accordingly reduced. 


\section{Intensity}

Since each proton will remain on average in the recirculator for $\frac{X_{I}}{x}$ turns, the beam intensity will be correspondingly increased. We give below the typical beam intensity to be considered, starting from $6 \times 10^{6}$ protons (i.e. $10 \mathrm{~mA}$ ), for a target length equal to $\beta^{\text {crit }}$.

\begin{tabular}{cccc}
\hline Mat. & $\rho\left(\mathrm{g} / \mathrm{cm}^{3}\right)$ & $X_{I}(\mathrm{~cm})$ & $I^{\text {equil. }}=I_{0} \cdot X_{I} / \beta^{\text {crit }}(\mathrm{A})$ \\
\hline \hline$D_{2}$ & 0.17 & 325 & 0.58 \\
\hline $\mathrm{H}_{2} \mathrm{O}$ & 1 & 85 & 0.85 \\
\hline $\mathrm{Be}$ & 1.85 & 40.5 & 0.19 \\
\hline $\mathrm{Pb}$ & 11.4 & 16.8 & 2.1 \\
\hline
\end{tabular}

Here beryllium which offers high density and low Z at the same time is the clear winner of the materials considered.

\section{Particle Capture}

It could be an advantage to handle a point-like source. In particular a pointlike source can be placed exactly in the focal point of an optical capture device such as a magnetic horn. A study in progress (3) to simulate a horn for the Neutrino Factory taking experience from the NGS studies (4) has already reached a performance similar to that obtained with a solenoid capture can be envisaged.

\section{Time Structure}

The favoured operation mode for the Neutrino Factory that emerged from NUFACT99 in Lyon would be pulsed operation with a repetition rate of 10$50 \mathrm{~Hz}$. Pulsed devices being operational for several hundredth of microseconds, and the recirculation time being of less or equal to a microsecond (recirculator ring of less than 300 meters circumference), there is plenty of time for the protons to recirculate from one or several initial bunches of protons injected in the system. If one starts from one bunch or train of bunches of protons, the recirculator generates then a succession of bunches (or trains) with a time structure equal to the recirculation time and with exponentially decreasing intensity $I_{k}=I_{\text {initial }} e^{-k \cdot x / X_{I}}$. 
Provided that the muon storage ring does not have a length that coincides with the recirculating time, it should be possible to store the successive muon bunches in the muon decay ring, provided an RF injection scheme is considered. This recirculator scheme could therefore be acceptable and offer some advantages for the Neutrino Factory. For Muon Colliders, evidently, the dilution of intensity over so many bunches would result in unacceptable loss of luminosity.

\section{Conclusions}

The possibility of recirculating the protons in a storage ring coupled with a thin target had been investigated. A thin target composed by low-Z material like $\mathrm{D}_{2}, \mathrm{H}_{2} \mathrm{O}$ or $\mathrm{Be}$ seems the best choice. The advantages of having a capture system like a magnetic horn, in particular for a thin target scheme, are still under investigation. The timing of the system seems at first look acceptable for a Neutrino Factory, but not for a Muon Collider.

\section{References}

[1] N. Mokhov and A. Van Ginneken, Increasing anti-proton yields via recirculating beam targeting, FERMILAB-FN-621 (1994).

[2] Particle Data Group, Euro. Phys. Journal C3 (1998) 76

[3] A. Ball, A. Blondel, S. Gilardoni, N. Vassilopoulos, Preliminary magnetic horn study for the collection scheme of a Neutrino Factory, to appear in the proceedings of NUFACT99, Lyon 1999.

[4] The CERN Neutrino beam to Gran Sasso (NGS), Addendum to report CERN 98-02, INFN/AE-98/05, CERN- SL/99-034(DI), INFN/AE$99 / 05$

[5] M. Vretenar in Prospective study of Muon Storage Ring at Cern, B. Autin, A. Blondel and J. Ellis eds, CERN 99-02 (1999) 8. 